Voix et Images

\title{
La poésie est mon athanor. Entretien avec Guy Lafond
}

\section{Thérèse Renaud}

Volume 4, numéro 2, décembre 1978

Guy Lafond

URI : https://id.erudit.org/iderudit/200148ar

DOI : https://doi.org/10.7202/200148ar

Aller au sommaire du numéro

Éditeur(s)

Les Presses de l'Université du Québec

ISSN

0318-9201 (imprimé)

1705-933X (numérique)

Découvrir la revue

Citer ce document

Renaud, T. (1978). La poésie est mon athanor. Entretien avec Guy Lafond. Voix et Images, 4(2), 179-186. https://doi.org/10.7202/200148ar d'utilisation que vous pouvez consulter en ligne.

https://apropos.erudit.org/fr/usagers/politique-dutilisation/ 


\section{La poésie est mon athanor Entretien avec Guy Lafond}

T. R. Quelle place accordez-vous à la poésie dans votre vie?

G. L. La poésie n'est pas à l'extérieur de ma vie, elle en fait partie comme un élément de recherche fondamentale. Je me considère comme alchimiste de la vie et la poésie est mon athanor. Ma poésie est donc une expérience tirée directement de la vie avec la certitude qu'elle doit servir ceux qui m'entourent. C'est dans ce sens que je ne me considère pas comme un poète à la manière de Rilke, qui mise sur la seule expérience poétique, bien que j'éprouve pour son cuvre une profonde estime. Je pratique avec la même passion d'autres disciplines, telles que le yoga, la musique, une certaine recherche métaphysique: toutes s'imbriquent dans une même poursuite avec l'impossibilité de fixer en moi des lieux privilégiés de connaissance. La poésie sert donc à unifier cet ensemble, c'est en elle qu'une synthèse s'effectue. Elle noue tout l'acquis de l'expérience en un faisceau, puis, par une sorte d'intuition archétypale, elle projette cette intuition rénovée vers de nouvelles difficultés. La poésie exerce donc un très grand pouvoir: celui d'affirmer cette connaissance et de la remettre en doute tout à la fois.

T. R. Comment vivez-vous ce rapport connaissance-vie-poésie?

G. L. Depuis ma plus tendre enfance je me sens créé par l'action et le regard des autres, à tel point que je perds tous mes moyens à la moindre manifestation de méfiance à mon égard. C'est dire combien j'attache d'importance à la fonction poétique, à ce qu'elle exerce sur les autres! II m'est impossible d'écrire ou de lire même, sans songer qu'un jour cela servira : j'éprouve la nécessité de cette conviction; sans elle je suis livré à une sorte d'apathie ou d'inertie. Bien entendu, je ne cherche pas à convaincre par des moyens didactiques, mais par une façon de vivre intensément les problèmes et, si tel est le cas, d'emblée ce vécu sera communiqué aux lecteurs; l'œuvre le transformera comme elle m'a renouvelé. Je n'écris pas pour éprouver une satisfaction personnelle, mais pour permettre à l'être d'évoluer. 
T. R. Avez-vous rencontré dans votre entourage familial ce ferment nécessaire aux poètes?

G. L. Si je remonte à mes origines, je constate que j'ai hérité de mon père une grande passivité vis-à-vis des événements de la vie, cependant qu'à l'intérieur de cette passivité je découvre une attention soutenue. Ce processus me servira à merveille dans l'exercice poétique, puisque la passivité me permettra de cultiver tout à la fois l'attente, la lente incubation du texte, en même temps que d'exercer mon regard critique. En somme, je pourrai peu à peu surveiller l'œuvre qui naît avec suffisamment d'attention pour qu'elle ne dévie pas.

Le sens artistique me vient de ma mère qui a fait des études musicales et nous a communiqué; dans une joie de vivre, sa recherche du beau, avec un regard global pour les choses. Ma mère m'a inculqué le sens de l'harmonie, elle qui aura souffert de ne pouvoir régler sa vie, et les circonstances de sa vie selon un ordre qu'elle ressentait, elle qui sera si fréquemment blessée par le moindre heurt, le moindre propos ou objet déplacé. Ma mère possédait l'art de l'observation perspicace, ce qui a développé très tôt chez le petit garçon que j'étais ce côté contemplatif qui favorisera, à la longue, certaines expériences dont celle de la poésie.

Jeune, je passais de longues heures dans un fauteuil, ou couché sur l'herbe ou sur la plage: je prenais le pouls du temps. J'attendais des jours entiers dans la plus grande passivité que certaines émotions, certaines pensées naissent ou se dissolvent. J'ai dû reconquérir cette disponibilité, une éducation trop portée vers l'efficacité m'en ayant éloigné en me faisant ressentir cette forme d'oisiveté comme culpabilisante! Je crois avoir découvert ce que représentait ces longues heures de cogitations solitaires. Plus tard, grâce au yoga je saurai mieux me servir de ces méditations.

Parce qu'il dissout la vision, l'instinct contemplatif m'a obligé très jeune à accorder une attention particulière aux questions formelles. Mes préoccupations se sont dissoutes pour ne conserver que celles ayant trait à l'émotion esthétique. Je dois à la musique d'avoir découvert le sens ou la nécessité de la forme. Celle-ci m'a convaincu de la richesse et de la valeur de l'art, et a déterminé chez moi le souci presque exclusif de la forme.

T. R. . Pouvez-vous élaborer pour nous ce qui constitue votre cheminement poétique?

G. L. Mon tout premier poème, écrit à l'âge de quinze ans, s'intitule Animus et Anima. A noter que je ne connaissais pas ces termes à travers Jung, mais à travers Claudel si ma mémoire est bonne. Ce poème de dix pages est révélateur de thèmes et de structures que je n'aurai de cesse de développer plus tard. II s'agissait d'un long dialogue sur le sens 
de la vie, de sa relation à la mort. Cette même relation de la vie à la mort constituera le fondement de mon œuvre poétique. Cette première poésie est donc très importante. Elle contient en germe le fondement de mon ceuvre poétique. A partir de ce moment, je tenterai de communiquer cette vision globale de la-vie et de la mort et cela à travers une forme qui désire immobiliser la vision.

En fait, ma poésie relève d'une longue méditation sur la mort. II s'agit de la mort non pas vécue comme rupture, mais imbriquée dans chaque geste ou chaque événement de la vie. En somme, je considère que la vie dans sa continuité n'est qu'un phénomène de mémoire, d'une certaine mémoire. En réalité (et cela, je l'ai vécu) il y a «entre» chaque quanta de vie, un quanta de mort, comme si la mort était un moteur de vie interférant dans chaque instant de vie pour la relancer.

Cela m'a amené, très tôt, à m'interroger sur la question du temps : question qui deviendra tragique par moments, à la limite de la sensation du néant. Ces questions ne peuvent être abordées que hors du temps, pour des raisons évidentes. Pour pénétrer ces zones où tout s'immobilise dans le regard, il faut une conscience unifiée, une forme d'objectivation. C'est sans doute parce que j'aborde ces lieux privilégiés qu'on qualifie ma poésie de désincarnée! Pour moi, elle participe aux moments les plus intenses de la vie. Ma poésie est réelle, elle doit être agissante, autrement elle perd son nom.

T. R. On peut donc considérer qu'à partir de votre prise de conscience, votre poésie se transforme aussi?

G. L. Si j'analyse l'évolution de mon œuvre poétique, je m'aperçois que dès mon premier livre J'ai choisi la mort, j'aborde le problème du détachement lié à la mort. Ce thème sera repris et développé dans Poèmes de l'Un, avec une recherche d'unité permettant l'accord des contraires. Dans le livre suivant, l'Eau ronde, je découvre que ce sens d'unité est un leurre, qu'il doit être dépassé; le poème gît dans un silence total, il débouche sur le néant. Les Cloches d'autres mondes, par contre, tente de franchir ce silence en l'apprivoisant.

La forme de mes livres est celle d'une symphonie, avec ses mouvements; elle cherche un mode circulaire d'expression, le livre se ferme comme il s'est ouvert. Cette forme sphérique sera davantage définie dans l'Eau ronde. Impossible ici de lire une ligne sans se référer au tout; le texte devient incompréhensible, ou une simple juxtaposition d'aphorismes sans cette référence. Dans mon prochain livre dont le titre m'est déjà donné : Vaincre le bleu, je tenterai de découvrir si de l'autre côté du silence il y a une parole.

Je suis comme tous les poètes, je voudrais pouvoir écrire «rose» et tout serait dit! 
T. R. Écrivez-vous par intermittence ou dans un geste continu?

G. L. Avant ma première publication J'ai choisi la mort, il y a un nombre incalculable de poèmes. Les uns sont autobiographiques, les autres s'attachent au mécanisme d'une structure, ou à une recherche formelle. A cette époque je pratiquais le sonnet plus particulièrement. En m'imposant des sujets, j'arrivais à en écrire deux ou trois par jour. J'éprouvais une grande satisfaction à l'exercice de cette virtuosité, mais je découvrais en même temps qu'il y a un très grand danger dans cette pratique; vous arrivez à manipuler des images, des rythmes, presqu'à volonté... mais vous vous y enfermez. Le jeu en est fascinant, vous êtes séduit par vos possibilités, mais comme le fait remarquer si justement Raymond Abellio, "vous cherchez des signes et non le sens". Vous restez accrochés au miroitement du poème, non à son pouvoir de connaissance. Autrement dit, vous fabriquez de la poésie, vous n'édifiez pas une ceuvre.

T. R. Pouvez-vous nous situer l'époque de ce travail poétique?

G. L. Cela se passait dans les années 1945-1950. Tous ces poèmes furent détruits à la suite de critiques avisées de François Hertel. II a joué un rôle primordial dans ma jeunesse. C'est lui qui m'a fait comprendre que le poème n'est pas la transposition d'un journal psychologique, mais un acte vécu dans la réflexion poétique, que la poésie doit être une expérience directe de ce vécu. Grâce à Hertel, j'allais découvrir l'œuvre de Mallarmé et de Valéry (j'éprouvais une telle fascination pour Mallarmé que j'ai appris par cœur toute son œuvre afin de mieux me l'assimiler!). J'allais découvrir aussi que la poésie peut soutenir une recherche métaphysique, que la pensée poétique possède un pouvoir de synthèse immédiate et révélatrice. Enfin, j'allais comprendre que le vécu de l'image peut se transformer en symbole et que cela est l'aspect dynamique d'une certaine connaissance.

Je comprends maintenant que le symbole est la pierre d'achoppement du poète. C'est pourquoi j'ai été amené à renoncer à la forme symbolique, car elle permet la satisfaction d'un inconnu non appréhendé cependant que vivement ressenti. Je crois qu'il faut posséder et maîtriser cet inconnu. S'il y a symbole, cela ne peut être que dans une définition spécifique du symbole, l'assimilant à la forme, et non plus à une «image " multidimensionnelle!

T. R. Votre poésie vous sert donc d'expérience immédiate?

G. L. Poür 'répondre à votre quéestion, je dirai que si je n'écris pas chäque jour (je dois attendre parfois assez longtemps pour recevoir l'intuition d'un poème ou d'un livre; cela se présente généralement d'un seul coup et alors je suis hanté par le texte à écrire), il n'y a pas d'expérience pour moi qui ne tente de s'articuler soit par l'image, soit par une synthèse des relations que crée cette expérience. Cela se vit dans une tension intolérable parfois. 
Nous sommes prisonniers de notre conscience sans possibilité de rompre le cercle vicieux dans lequel elle nous enferme. II n'y a de connaissance que par la relation «sujet-objet". Si j'arrive à objectiver la conscience, je reste malgré tout sous son emprise par la nécessité de subir sa loi. Comment connaître alors la vérité, son origine, sinon par référence à quelque néant insondable? J'ai longtemps vécu ce problème. Dans un premier temps, j'ai tenté d'objectiver ce problème de conscience; je fus saisi par l'intime relativité des choses, par ces relations universelles qui les lient entre elles. Ainsi, je ne peux pénétrer dans une chambre sans qu'immédiatement tous les objets de cette chambre deviennent des énigmes, et qu'apparaisse la relation avec chacun d'eux, avec l'ensemble de la chambre, avec moi-même. C'est hallucinant!

Je pense aujourd'hui avoir pris mes distances avec cette dialectique crucifiante. Pour effectuer ce dépassement, je m'appuie sur les textes de ceux qui ont parcouru ce chemin.

T.R. Que devient la musique dans votre prise de conscience créatrice?

G. L. Vous avez raison de me poser cette question, parce qu'en fait c'est le nœud de ma vie. C'est par la musique que je m'exprime le mieux, mais hélas, malgré les circonstances du jeune âge où nous faisions de la musique en famille, ce n'est qu'après avoir terminé le cours classique que j'ai pu aborder sérieusement mes études musicales. De ce fait, la musique n'est jamais devenue un langage direct, je ne peux m'exprimer entièrement par elle. Pourtant, je ne dirai jamais assez avec quel amour, avec quelle passion j'ai servi la musique! Je suis donc devenu interprète pour moñ propre contentement. Dernièrement, j'ai décidé de rompre avec cette forme de travail; l'interprétation musicale me donnant trop de satisfaction, me vidait de toute rigueur envers moi-même. J'ai horreur de m'installer quelque part, je veux que la vie soit un chemin perpétuel. Je crois aux questions, non aux réponses.

II n'empêche que pour moi, la musique marque l'aboutissement naturel de la poésie. Qui a dit : «Tout poème aspire à devenir musique»? Je le crois volontiers, dans la définition la plus haute de la musique : l'art qui crée le temps. Ainsi la musique tente, par le son, d'incarner le temps comme la poésie cherche, par le langage, le mot, la forme, à manifester l'idée. Lorsque j'ai abordé la poésie, j'ai immédiatement cherché à développer des formes musicales. C'est en étudiant Beethoven que la forme linéaire de J'ai choisi la mort m'a été suggérée.

J'aimerais assez aborder le problème de l'Eau ronde qui m'a été imposé presque d'une façon osmotique. On a parlé de ce livre comme de quelque chose d'incompréhensible, d'un recueil d'aphorismes. Dans ce livre, le poème n'est jamais dévoilé, je le dis expressément à plusieurs endroits. J'ai voulu cerner une forme spécifique de silence, afin 
que naisse spontanément chez le lecteur le chant ou le poème de ce silence particulier. C'est par le jeu créé par la superposition de ces mêmes aphorismes que je suscite l'intérêt, en donnant à lire entre les lignes. Je voudrais que le lecteur saisisse les niveaux de pensées, le mouvement, entre les lignes, entre les mots, entre les aphorismes, pour que d'un seul coup surgisse en lui ce poème non écrit, qui est cependant déterminé : que naisse en lui, spontanément la saveur du silence voulu. C'est la recherche d'une poésie “mantrique», celle qui donne l'objet immédiatement; c'est aussi une forme issue de certaines recherches en musique contemporaine, où l'on tente de créer un temps musical en le parcourant sous toutes ses facettes sans jamais qu'il ne s'articule. C'est là un des aspects de ce livre. L'Eau ronde parcouru par un regard purement littéraire n'a aucun sens.

T. R. Avez-vous subi des influences importantes sur le plan poétique?

G. L. Je dirais que j'adore les influences, mais je sens qu'il y a dans cette affirmation le besoin de contredire ceux qui les craignent! J'aime chercher cette œuvre nouvelle qui influencera ma vision, je tente de m'en imprégner au point d'habiter le cerveau ou le cœur de celui qui l'a écrite! Les influences acceptées nous libèrent plus rapidement et de façon plus lucide que ne le ferait un regard porté uniquement sur soimême. On peut lire partout des influences dans mes textes, je ne chercherai jamais à les dissimuler, j'en suis même fier. Non seulement elles fortifient ma propre attitude poétique, mais elles libèrent tout un matériel qui mettrait des années à monter à la surface. Elles m'assurent d'une continuité dans le corps poétique.

Mallarmé fut mon premier maître. J'ai analysé pendant des mois "l'Après-midi d'un faune", un peu comme on fait l'analyse formelle d'une œuvre musicale, pour en percer tous les mystères, toute la profondeur d'expression, la coloration du mot, le rythme des vers, inhérents à la symbolique mallarméenne.

Je découvrirai par la suite chez Valéry, et surtout dans "la Jeune Parque ", le pouvoir créateur du poète. Quel poème sublime où tout à la fois le matin, la lumière, l'inspiration se lèvent comme la jeune Parque s'éveille. Aucun mot, aucun rythme maladroit ne viennent détourner de la sensation même du matin, de la lumière, de la pensée... Vous terminez la lecture aussi étincelant que le poème, marqué par l'émotion qu'apporte la maîtrise, le contrôle souverain du langage. J'en frémis encore!

Avec Rilke, j'ai découvert la splendeur du lyrisme. C'est le cœur qui s'ouvre et dévoile son chant propre, porteur d'une forme d'intuition particulière qu'on peut appeler, sans la définir, «psychique».

J'ai beaucoup lu les poètes américains, parce que chez eux je sens une technique de l'écriture poétique très ferme, très définie, portée à 
l'ultime limite. Après Ezra Pound et Eliott, E. E. Cummings m'a beaucoup influencé. Sa volonté de rompre avec la syntaxe habituelle, son désir de pénétrer au cœur du mot pour lui permettre de révéler tout son contenu, m'a profondément marqué.

Puis, vous m'avez demandé de lire le Livre des questions d'Edmond Jabès. Je cherchais à ce moment la forme à donner à mon inspiration, à mon écriture. J'étais dans le noir le plus total. Tout à coup, il m'a semblé découvrir chez Jabès, cette forme qui se cherchait en moi. Nous nous sommes fréquentés pendant deux années, le temps d'écrire l'Eau ronde. J'ai reçu là, ce que j'avais reçu d'autres maîtres en d'autres domaines, un enseignement par influence directe. II parlait peu et toujours très simplement. Des remarques dans le style: "Vous ne creusez pas assez vos images "... Parfois il raturait un vers... II m'accusera d'impatience! Tous ces dialogues firent disparaître mes scrupules littéraires. Je pris conscience, à travers cette fréquentation, du sérieux dans le travail de la matière poétique. Oui, Jabès m'a libéré...

Longtemps j'ai été séduit par René Char et Saint-John Perse. D'autres poètes ont pris la relève: Gottfried Benn, Trakl, Celan, Mandelstam, mais surtout Guillen qui me donne envie d'une poésie simple, directe, en accord avec ce que je sens comme évolution normale de mon écriture.

II faut ajouter à ce florilège, des influences diverses comme celles des Raymond Abellio, Heidegger, Nietzsche, Sri Aurobindo, pour ne nommer que les plus importantes et cela à travers leurs écrits.

C'est dans un rapport plus direct que j'ai subi une toute autre forme d'influence, celle de Sivananda et de Mère. J'ai rencontré Mère au moment où je tentais de me dégager du symbolisme. Pour qui connaît l'influence qu'exerce un maître spirituel, celui-ci comprendra que le personnage de Mère soit chargé de symboles. J'étais lié incompréhensiblement, je tentais vainement de me défaire de cette emprise. Puis Mère meurt. A l'annonce de son départ je ne me sens nullement attristé, mais comme intimement soulagé par la disparition de cette image. Je suis en face d'une autre réalité. Étrange sensation où tout le ciel s'ouvre devant soi, où le mouvement réel de la conscience apparaît clairement. Aucune image, aucun symbole n'exerceront de séduction dorénavant. D'un coup, je suis libéré et prêt à me servir de ces nouvelles significations pour leurs valeurs intrinsèques.

J'ai choisi la mort est issu directement de mon passage chez Sivananda, où j'ai vécu l'expérience de la paix, de son imprégnation la plus totale. Puis Poèmes de l'Un, toujours sous l'emprise du symbolisme, portera cette Présence dévoilée vers une vision d'unité. C'est à ce moment que je commençai l'étude de l'œuvre de Jung qui me fut révélatrice. 
Chaque poète résoud à sa manière le problème du symbolisme, ou tout au moins celui de la métaphore. Le symbole est un vécu, non une donnée littéraire. Il oblige le mot à déborder de ses limites. Chaque fois que je me servirai du mot "rose" hors de sa structure intime, je déborderai la limite du mot, donc celle du poème.

La saveur de la pomme se révèle par le goût, non par une analyse mentale, sans quoi éternellement la saveur de la pomme demeurera un mystère! La connaissance est toujours sous le signe de l'évidence. Ainsi le mot, le poème. Le lecteur qui cherchera chez moi un sens trop unilatéralement philosophique se perdra dans un vain hermétisme. On ne connaît, on n'écrit que par le corps. Encore faut-il élargir un peu la notion de corps...

T. R. Vous donnez cependant l'impression d'un déracinement.

G. L. Écrire avec ses racines est un faux problème, comme si l'arbre pouvait vivre en dehors de lui-même! Je dis que l'écriture est un phénomène physique, que l'on écrit avec le corps, que l'on pense avec le corps. Je me suis interrogé sur ces questions de racines très tôt parce que, étant né en Ontario, je me suis senti aliéné très longtemps, ne pouvant d'aucune manière savoir à qui et à quoi j'appartenais. Dans un essai qui n'a pas été publié, je situe de façon très précise le lieu de ces racines, mais il serait trop long ici d'entrer dans ces détails. Depuis, le problème est résolu pour moi, je n'écris pas avec mes racines, mais à l'intérieur du fruit fécondé par ces mêmes racines. Ce qui m'intéresse, c'est de connaître le fruit nouveau que porteront ces racines!

T. R. Que pensez-vous du Québec actuel?

G. L. Je sens le Québec très fortement en moi, parfois comme un bienêtre, parfois comme un rhumatisme. Il me nourrit et il me tue, à la fois. Mais lui seul me permet la reconnaissance de la totalité de mon être. II est dans mon sang pour le meilleur et pour le pire, et je n'écris pas hors du sang... Je trouve qu'on méconnaît le champ d'expérience qu'il pourrait être, l'immense réservoir de créativité qu'il recèle, et que les projets que l'on nourrit à son endroit sont bien souvent étriqués. Mais tout cela devrait être plus nuancé. II reste que j'ai la sensation de participer par l'exercice de ma poésie à l'évolution présente du Québec. Ce même Québec si difficile à convaincre de la valeur efficace de la poésie! Je suis d'une certaine façon un solitaire, mais un solitaire qui n'a jamais perdu le sens de la solidarité.

(Entrevue réalisée par Thérèse Renaud.) 